\title{
Intervenção Coronária em Hospital Geral no Piauí
}

\author{
Antenor Portela, Carlos Linhares, Raldir Bastos, Jayro Paiva
}

Teresina, PI

\begin{abstract}
Objetivo - Determinar o índice de sucesso e complicações das intervenções coronárias realizadas em hospital geral do Piauí, em um laboratório de hemodinâmica com baixo volume de procedimentos por ano.

Métodos-Cento e vinte e dois pacientes, submetidos a 146 procedimentos de intervenção coronária, de agosto/91 a janeiro/98 tiveram seus dados clínicos e angiográficos analisados retrospectivamente. As cineangiocoronariografias e as intervenções foram realizadas em aparelho com sistema de fluoroscopia com câmara e monitor de TV de 525 linhas. As variáveis analisadas foram o sucesso inicial do procedimento (estenose residual $<50 \%$ e fluxo TIMI III) e as complicações maiores (oclusão aguda, infarto, cirurgia de urgência ébito). Foram revisados os dados clínicos e as características morfológicas das lesões (ACC/AHA Task Force).

Resultados- $A$ média etária foi de 61 anos, variando de 25 a 85, 67\% dos pacientes eram do sexo masculino, 85\% apresentavam síndrome isquêmica aguda (infarto agudo do miocárdio, angina pós infarto ou angina de repouso), 5\% apresentavam-se em choque cardiogênico. $88 \%$ das lesões eram tipo B. O índice de sucesso imediato foi de 93\%. A taxa de complicações maiores (oclusão aguda, cirurgia de urgência, infarto e óbito) foi de 3,5\%.

Conclusão - A despeito de um baixo volume de procedimentos por ano e um equipamento de cineangiocoronariografia convencional, o índice de sucesso desta instituição foi excelente em uma população de pacientes diversa e complexa com taxa de complicações semelhante à encontrada na literatura.
\end{abstract}

Palavras-chaves: doença arterial coronária, cardiologia intervencionista, angioplatia coronária

\section{Coronary Intervention Perfomed in a General Hospital in the State of Piauí}

Purpose - To evaluate retrospectively success and complication rates of coronary interventions performed in a general hospital with no digital equipment in the State of Piaui with a small annual number of procedures.

Methods - From August/91 to January/98, 122 patients underwent 146 procedures using a fluoroscope with camera and TV monitor of 525 lines. The variables analyzed included the initial success of procedures (residual stenosis less than fifty percent and TIMI III flow) and more serious complications (acute occlusion, infarcts, emergency surgeries and death). The clinical data and the morphological characteristics of the lesions (ACC/AHA Task Force) were also analyzed.

Results - Eighty five percent of the patients presented with an acute ischemic syndrome (acute myocardial infarction, post infarction angina, rest angina), 5\% were in cardiogenic shock and $88 \%$ had type $B$ lesions. The initial success rate was $93 \%$ while the more serious complications occurred in $3.5 \%$ of the cases.

Conclusion - In spite of the small annual procedural volume and the use of conventional equipment, the initial success rate in our institution was excellent in a diverse and complex patient population while the complication rates were similar to that of the literature.

Key-words: coronary artery disease, interventional cardiology, coronary angioplasty

Arq Bras Cardiol, volume 71 (n 4), 591-594, 1998

O desenvolvimento da cardiologia intervencionista tem possibilitado que um número maior de pacientes e com maior grau de complexidade seja abordado de forma não

Hospital São Marcos - Teresina

Correspondência: Antenor Portela - Rua Desembargador Pires Castro, 584-S 64001-390 - Teresina, PI

Recebido para publicação em 23/4/98

Aceito em 1/7/98 cirúrgica, notadamente na doença arterial coronária. Este fato tem contribuído para o surgimento de novos serviços que realizam tais procedimentos e, como consequiência, um volume menor de procedimento por serviço. A comunidade científica internacional tem demonstrado preocupação com este fato $^{1,2}$ e recomendações rigorosas para um volume mínimo de procedimentos por ano por operador têm sido feitas ${ }^{3}$. Estas indagações surgiram a partir de relatos mostrando que a morbidade e mortalidade nas cirurgias de revascularização 
do miocárdio, eram tanto maiores quanto menor o volume de cirurgias realizadas pelo cirurgião ${ }^{4}$. Da mesma forma, um maior índice de complicações em angioplastia coronária realizada em um laboratório com um número de procedimentos reduzido também foi relatado ${ }^{5}$.

A Sociedade Brasileira de Cardiologia nas suas Diretrizes sobre Angioplastia Transluminal Coronária ${ }^{6}$ recomenda que para execução de terapêutica intervencionista o laboratório de hemodinâmica possua aparelho de raio X com sistema de fluoroscopia que deverá trabalhar com câmara e monitor de televisão de 1024 linhas

Em um país de extensa dimensão como o Brasil, o acesso a tratamento médico de alta complexidade se deu à custa da proliferação dos serviços prestadores, com volume anual de procedimentos conseqüentemente mais baixo por serviço. Por esta razão, questionamentos sobre a segurança da realização de intervenções coronárias são pertinentes ${ }^{7,8}$.

Este trabalho foi realizado para determinar o índice de sucesso e complicações das intervenções coronárias realizadas em laboratório de hemodinâmica com baixo volume de procedimentos por ano, em Hospital Geral, no Piauí, utilizando um equipamento de hemodinâmica convencional (não digital, com resolução de 525 linhas).

\section{Métodos}

De agosto/91 a janeiro/98, foram realizados 146 procedimentos de intervenção coronária em 122 pacientes consecutivos no Serviço de Hemodinâmica do Hospital São Marcos em Teresina, de acordo com indicação clínica do médico assistente e avaliação prévia da equipe de hemodinâmica. Os dados clínicos e angiográficos de todos os pacientes foram analisados, retrospectivamente. Foram incluídos todos os pacientes que realizaram angioplastia ou implante de stent neste período.

As cineangiocoronariografias e as intervenções foram realizadas em um único aparelho, utilizando fluoroscopia com câmara e monitor de televisão de 525 linhas (Angioskop D33 - Siemens). Todos os pacientes receberam 10.000U de heparina intra arterial após inserção da bainha vascular na artéria femoral ou introdução do cateter guia em artéria braquial, sendo complementada a anticoagulação, quando necessária, para manter o tempo de coagulação ativado acima de 300s e foram mantidos com ácido acetilsalicílico 100mg por dia, indefinidamente, quando submetidos à angioplastia com balão e com associação de ácido acetilsalicílico e ticlopidina ( $500 \mathrm{mg} / \mathrm{dia}$ ) por um mês e, posteriormente, apenas ácido acetilsalicílico $100 \mathrm{mg}$ por dia, se submetidos a implante de stent. Durante a internação hospitalar os pacientes foram examinados diariamente por um dos autores do trabalho e tiveram seus dados clínicos registrados em prontuário. Todos os pacientes realizaram eletrocardiograma (ECG) diariamente até o $3^{\circ}$ dia após a angioplastia e no dia da alta hospitalar, conforme protocolo do serviço de hemodinâmica. Exames adicionais eram solicitados conforme indicação do médico assistente.
As variáveis avaliadas foram o sucesso inicial do procedimento, definido como estenose residual $<50 \%$ e fluxo anterógrado TIMI III, ou seja, opacificação completa do leito coronário distal até o $3^{\circ}$ ciclo cardíaco ${ }^{9}$, e o surgimento de complicações: 1) óbito, ocorrido em qualquer momento durante a internação relacionada ao procedimento; 2) infarto, diagnosticado pelo surgimento de nova onda Q no ECG; 3) oclusão aguda, identificada pelo surgimento de dor torácica típica ou alteração eletrocardiográfica ou ambas que resultaram no retorno do paciente ao laboratório de hemodinâmica, computada somente quando não houve outra complicação maior; 4) cirurgia de revascularização durante a internação.

Foi realizada revisão de prontuários e os pacientes classificados de acordo com sua condição clínica em: portadores de infarto agudo do miocárdio (IAM), quando apresentavam dor torácica típica e alteração eletrocardiográfica compatível com infarto com onda Q; angina pós infarto, quando havia dor torácica típica e diagnóstico recente de infarto; angina instável, quando apresentavam dor torácica típica em repouso ou angina progredida na última semana; angina estável quando não havia relato de mudança recente no quadro anginoso.

As características morfológicas das lesões foram avaliadas utilizando-se os critérios do American Heart Association Task Forcel American College of Cardiology ${ }^{3} \mathrm{e}$ modificados por Ellis e $\mathrm{col}^{8}$. A classificação definitiva da lesão foi dada após consenso entre os analisadores.

Os índices de sucesso e complicações foram comparados com aqueles informados pela Central Nacional de Intervenções Cardiovasculares (CENIC) ${ }^{10}$.

Os resultados foram expressos como proporção ou média \pm desvio padrão. As diferenças entre proporções de categorias foram analisadas com o teste do qui-quadrado.

\section{Resultados}

Foram realizados 146 procedimentos em 122 pacientes e realizadas $133(91 \%)$ angioplastias com balão e $13(9 \%)$ implantes de stent. Os dados epidemiológicos, as características clínicas dos pacientes e a morfologia das lesões estão apresentados na tabela 1. A média etária foi de 61 anos, variando de 25 a 85 anos, sendo que $67 \%$ dos pacientes eram do sexo masculino. $85 \%$ apresentavam síndrome isquêmica aguda (IAM, angina pós infarto e angina de repouso). Seis (5\%) pacientes com síndrome isquêmica aguda tinham o diagnóstico clínico de choque cardiogênico. A análise da morfologia das lesões mostrou complexidade intermediária em $88 \%$ dos casos (morfologias B1 e B2).

O índice de sucesso imediato foi de 93\% (136 procedimentos para um total de 146). A taxa de complicações hospitalares graves (infarto Q e cirurgia de revascularização de urgência) calculada por procedimento foi de $3,5 \%$, sendo que 2 pacientes foram submetidos à cirurgia de revascularização por dissecção extensa do vaso tratado. Dois pacientes cursaram com surgimento de onda $Q$ no ECG, porém sem dor típica ou instabilidade hemodinâmica e tiveram boa evolução clínica. Um paciente apresentou dor torácica típica, 


\begin{tabular}{|lcc|}
\hline \multicolumn{2}{|c|}{$\begin{array}{c}\text { Tabela I - Dados epidemiológicos, características clínicas e } \\
\text { morfológicas das lesões coronárias em 122 pacientes }\end{array}$} \\
\hline A - Dados epidemiológicos & $\mathrm{N}$ & $\%$ \\
\hline Número & 122 & 100 \\
Idade média $=61 \pm 9$ (25-85) & 82 & 67 \\
Sexo masculino & 80 & 33 \\
\hline Sexo feminino & 40 & \\
\hline B - Características clínicas & & 35 \\
Angina instável & 43 & 26 \\
Infarto agudo do miocárdio & 32 & 24 \\
Angina pós-infarto & 29 & 15 \\
Angina estável & 18 & \\
\hline C - Morfologia das lesões & & 46,7 \\
\hline Tipo B1 & & 41,0 \\
Tipo B2 & 57 & 6,6 \\
Tipo C & 50 & 5,7 \\
Tipo A & 8 & \\
\hline
\end{tabular}

\begin{tabular}{|lc|}
\hline $\begin{array}{l}\text { Tabela II - Complicações maiores em 146 procedimentos de } \\
\text { intervenção coronária realizados no Hospital São Marcos - } \\
\text { Teresina - Piauí de 1991 a 1998 }\end{array}$ \\
\hline \multicolumn{2}{|c|}{$\mathrm{N}(\%)$} \\
Complicação & $2(1,4)$ \\
Cirurgia de revascularização & $2(1,4)$ \\
Infarto Q & $1(0,7)$ \\
Oclusão aguda & 0 \\
Óbito & $5(3,5)$ \\
Total & \\
\hline
\end{tabular}

\begin{tabular}{|lcc|}
\hline $\begin{array}{c}\text { Tabela III - Comparação do índice de sucesso primário e } \\
\text { complicações, com os do Relatório do Registro Nacional - Central } \\
\text { Nacional de Intervenções Cardiovasculares (CENIC) }\end{array}$ \\
\hline & Sucesso \% & Complicações \% \\
\hline CENIC & 89,7 & 5 \\
Resultado obtido & 93 & 3,5 \\
$\mathrm{p}$ & 0,29 & 0,38 \\
\hline
\end{tabular}

$12 \mathrm{~h}$ após o procedimento e a cineangiocoronariografia revelou artéria ocluída com circulação colateral exuberante, evoluindo com infarto sem onda Q no ECG (tab. II). Não foram registrados óbitos durante a internação hospitalar relativos ao procedimento.
Os índices de sucesso imediato e complicações obtidos neste trabalho e os relatados pela CENIC ${ }^{10}$, estão apresentados na tabela III.

\section{Discussão}

O progresso e o desenvolvimento da cardiologia intervencionista têm ocorrido com enorme velocidade na última década, resultando na difusão de novos métodos de intervenção coronária em todo o país e tornando possível o acesso da população a procedimentos de alta complexidade nas regiões mais distantes dos grandes centros da cardiologia nacional.

Neste Hospital Geral, com baixo volume de procedimentos de intervenção coronária por ano ${ }^{11}$, utilizando-se de equipamento de cineangiocoronariografia convencional, o sucesso dos procedimentos de intervenção em um período de seis anos foi equivalente, se não superior, aos resultados publicados pela CENIC $^{10}$.

Nesta série de 122 pacientes, observam-se aspectos clínicos e angiográficos que os caracterizam como doentes coronários de moderada complexidade, a julgar pela idade média de 61 anos, pacientes do sexo feminino em $33 \%$ dos caso, portadores de síndrome isquêmica aguda em $85 \%$ dos casos, em particular infarto em evolução em $26 \%$ e lesões do tipo B em $73 \%$. A despeito da complexidade clínica e angiográfica, o sucesso primário foi elevado (93\%), semelhante ao relatado na literatura ${ }^{7}$. As complicações maiores, incluindo infarto, cirurgia de emergência e óbito foram 1,4; 1,$4 ; 0 \%$, respectivamente. Estes dados são semelhantes aos informados pela CENIC ${ }^{10}$, apesar de na presente casuística, um número maior de casos agudos estar presente, o que poderia acarretar um índice inferior de sucesso ${ }^{12}$.

A seleção dos casos está provavelmente relacionada a uma maior disponibilidade dos serviços de cirurgia, para os quais a maioria dos pacientes eletivos é encaminhada.

Esses bons resultados estão relacionados a uma série de fatores, entre os quais citam-se: a seleção dos casos, excluindo-se lesões de alta complexidade (tipo C), experiência do operador e suporte de pessoal de enfermagem.

Conseguiu-se demonstrar nível de desempenho ótimo, por ano, em um serviço de baixo volume de procedimentos, demonstrado pela eficácia e segurança dos procedimentos.

\section{Referências}

1. Ellis SG, Omoigui N, Bittl JA et al - Analysis and comparison of operator-specific outcomes in interventional cardiology. Circulation 1996; 93: 431-9.

2. Kimmel SE, Berlin JA, Laskey WK - The relationship between coronary angioplasty procedure volume and major complications. JAMA 1995; 274: 1137-42.

3. Kyan TJ, Faxon DP, Gunnar RM et al - Guidelines for percutaneous transluminal angioplasty. A report of the American College of Cardiology/ American Heart Association task force on assessment of diagnostic and therapeutic cardiovascular procedure (subcommittee on percutaneous transluminal angioplasty). Circulation 1988; 78: 486-502.
4. Showstack JA, Rosenfeld KE, Garnick DW, Luft HS, Schaffarzick RW, Fowles T - Association of volume with outcome of coronary artery bypass graft surgery: schedule vs non-scheduled operations. JAMA 1987; 257: 785-9.

5. Jollis JG, Peterson ED, Delong ER et al - The relation between the volume of coronary angioplasty procedures at hospitals treating medicale beneficiaries and short term mortality. N Engl J Med 1994; 331: 1625-9.

6. Sociedade Brasileira de Cardiologia - Diretrizes da Sociedade Brasileira de Cardiologia sobre angioplastia transluminal percutânea. Arq Bras Cardiol 1995; 64: 489-500.

7. Klein LW, Schaer GL, Calvin JE et al - Does low individual operator coronary 
interventional procedural volume correlate with worse institutional procedural outcome? J Am Coll Cardiol 1997; 30: 870-7.

8. Ellis SG, Vandomaer MG, Couley MJ et al - Multivessel angioplasty study group: Coronary morphologic and clinical determinants of procedure outcomes with angioplasty for multivessel disease. Circulation 1990; 82: 1193-202.

9. The TIMI Study Group - The thrombolysis in myocardial infarction (TIMI) trial: Phase 1 findings. N Engl J Med 1985; 312: 932-6.

10. Sousa AGMR - Procedimentos percutâneos de intervenção cardiovascular no
Brasil em 1992 e 1993. Relatório do Registro Nacional - Central Nacional de Intervenções Cardiovasculares (CENIC). Arq Bras Cardiol 1994; 62: 217-23.

11. Hannan EL, Raez M, Ryan TJ et al - Coronary angioplasty volume-outcome relationships for hospital and cardiologists. JAMA 1997; 279: 892-8.

12. The TIMI Study Group - Comparison on invasive and conservative strategies following tissue plasminogen activator in acute myocardial infarction. Results of the thrombolysis in myocardial infarction (TIMI II) trial. N Engl J Med 1989; 320: 618-26. 